\title{
MULTI-SENSOR MEASUREMENTS OF MIXED-PHASE CLOUDS ABOVE GREENLAND
}

\author{
Robert A. Stillwell ${ }^{1 *}$, Matthew D. Shupe ${ }^{2,3}$, Jeffrey P. Thayer ${ }^{1}$, Ryan R. Neely III ${ }^{3,4}$, and David D. \\ Turner ${ }^{2}$
}

${ }^{1}$ Aerospace Engineering Sciences, University of Colorado, *Presenting Author, ${ }^{2}$ NOAA/Earth System Research Laboratory, Boulder, Colorado, ${ }^{3}$ Cooperative Institute for Research in Environmental Science, University of Colorado, ${ }^{4}$ School of Earth and Environment, University of Leeds

\begin{abstract}
Liquid-only and mixed-phase clouds in the Arctic strongly affect the regional surface energy and ice mass budgets, yet much remains unknown about the nature of these clouds due to the lack of intensive measurements. Lidar measurements of these clouds are challenged by very large signal dynamic range, which makes even seemingly simple tasks, such as thermodynamic phase classification, difficult. This work focuses on a set of measurements made by the Clouds Aerosol Polarization and Backscatter Lidar at Summit, Greenland and its retrieval algorithms, which use both analog and photon counting as well as orthogonal and non-orthogonal polarization retrievals to extend dynamic range and improve overall measurement quality and quantity. Presented here is an algorithm for cloud parameter retrievals that leverages enhanced dynamic range retrievals to classify mixed-phase clouds. This best guess retrieval is compared to co-located instruments for validation.
\end{abstract}

\section{INTRODUCTION}

Understanding the occurrence of mixed-phase clouds in the Arctic is a critical building block to better specifying regional energy and ice mass budgets [1]. Approximately 7 meters of sea-level rise is locked within the Greenland Ice Sheet (GrIS) of which approximately $25 \mathrm{~mm}$ has been contributed since 1900 with increasing rate in recent years $[2,3]$. Several studies have directly linked melting to changes in surface energy budgets caused by low level liquid-only and mixed-phase (here collectively referred to as mixed-phase) clouds [4,5]. The colloidally unstable mixture of liquid and ice water within clouds is a regular occurrence in the Arctic with a complicated set of processes serving to maintain both phases [6]. In the Arctic, the liquid phase dominates the radiative contribution of clouds while the ice phase modulates the liquid via the Wegener-Bergeron-Findeisen process $[7,8]$.
As a result of the importance of mixed-phase clouds to the surface energy and mass budgets of the GrIS, quantitative measurements of cloud properties are sought to better understand the processes driving and underlying the formation and persistence of these clouds. Historically, the unique and harsh environment of the Arctic has hampered such measurements. This work will focus on a unique set of continuous measurements made by lidar and other sensors centered at the top of the GrIS at Summit, Greenland $\left(72^{\circ} 35^{\prime} 46^{\prime \prime}\right.$ $\mathrm{N} ; 38^{\circ} 25^{\prime} 1^{\prime \prime} \mathrm{W}$; $3212 \mathrm{~m}$ asl) since 2010 as part of the Integrated Characterization of Energy Clouds Atmospheric State and Precipitation at Summit (ICECAPS) experiment [1]. Of interest for this contribution are co-located lidars, one custom polarization lidar and one commercially available polarization sensitive micropulse lidar (MPL), a single polarization Ka-band Doppler radar, and 2 microwave radiometers with measurement bands spanning 23 to $150 \mathrm{GHz}$.

\section{CLOUDS AEROSOL POLARIZATION AND BACKSCATTER LIDAR}

The Clouds Aerosol Polarization and Backscatter Lidar (CAPABL) has been deployed to Summit since 2010 to measure cloud phase and horizontally oriented ice crystals (HOIC) using polarization retrievals. The retrieval of HOIC by diattenuation measurements requires a minimum of 3 polarization channels in an off zenith orientation; CAPABL makes 4 linear polarization measurements allowing for orthogonal and nonorthogonal retrievals of polarization components [9]. This set of measurements is evenly spaced in signal intensity to more judiciously account for systematic signal saturation effects. The result of orthogonal and non-orthogonal retrievals is an expanded signal dynamic range [10].

This expanded signal dynamic range is helpful in making quantitative measurements of mixedphase clouds. Mixed-phase clouds occur predominantly low in the atmosphere (below 2-3 
$\mathrm{km}$ above the surface) at Summit, resulting in very high dynamic range signals, often well in excess of 5 orders of magnitude in a single profile. These differences in dynamic range caused by signal saturation create tremendous variability in estimates of fractional occurrence of cloud types, resulting in errors in excess of $30-40 \%$ [10]. These errors in fractional occurrence dramatically affect the interpretation of cloud radiative effects at Summit and cause yet unquantified uncertainty in cloud radiative forcing estimates.

The focus of this work is in developing a best estimate in cloud product to identify liquid, ice, and HOIC cloud types and to verify that best estimated product (hereafter referred to as data mask) with co-located sensors.

\section{CO-LOCATED SENSOR SUITE}

The ICECAPS sensor suite consists of many remote sensing instruments as well as a twicedaily radiosonde program modeled on the Atmospheric Radiation Measurement (ARM) sites [11]. The following will describe the relevant instruments and data comparison in greater detail.

\subsection{MICROPULSE LIDAR}

Multi-sensor comparisons of data are complicated by the designed sensitivities of instrumentation. It is difficult to compare different instruments, for example radar and lidar, because they are designed to perform different tasks. It is therefore reasonable to make a first comparison between two lidar systems. CAPABL is compared to a Sigma Space V4 (MPL) [12]. Relevant specifications are given in Table 1.

Table 1: Relevant lidar specifications. Note that CAPABL uses receiver attenuation filters to better observe the troposphere and makes the power aperture product of CAPABL less than the MPL by a factor of $\sim 50$.

\begin{tabular}{|l|c|c|}
\hline & CAPABL & MPL \\
\hline Laser Power [W] & 0.3 & 0.02 \\
\hline Receiver Attenuation [OD] & 3 & 0 \\
\hline Telescope Diameter [mm] & 208 & 178 \\
\hline Polarizations & 4 & 2 \\
\hline Range Resolution [m] & 25.98 & 30 \\
\hline Polarization Scan Res [sec] & $\sim 82$ & 80 \\
\hline
\end{tabular}

CAPABL and the MPL are forced to similar resolutions in post processing. Polarization retrievals and a Klett inversion are performed. MPL data is then interpolated in time and space to the CAPABL grid. Data is then classified via the scheme in [10] from 0 to $8 \mathrm{~km}$.

For this comparison, pixels are either classified as clear air, cloud liquid, cloud ice, or missing because of data filtering for each system. This allows 16 possible combinations of data from CAPABL and the MPL. An example of the data overlap is given in Table 2 for July 2016 where it is noted that each column is summed and normalized. Therefore each value is the percentage of CAPABL pixels of each type.

Table 2: Pixel by pixel comparison of CAPABL (C.) and the MPL for July 2016. The green/dotted color shows agreement. Red indicates one instrument had data removed by quality control steps. Yellow indicates enhanced sensitivity by CAPABL and gray enhanced sensitivity by the MPL. Purple indicates both instruments lack data.

\begin{tabular}{|c|c|c|c|c|}
\hline MPL & Clear & Liquid & Ice & Filtered \\
\hline Clear & $69.7 \%$ & $37.0 \%$ & $62.2 \%$ & $3.42 \%$ \\
\hline Liquid & $0.3 \%$ & $56.3 \%$ & $5.5 \%$ & $0.08 \%$ \\
\hline Ice & $0.2 \%$ & $3.7 \%$ & $29.4 \%$ & $0.50 \%$ \\
\hline Filtered & $29.9 \%$ & $3.0 \%$ & $3.02 \%$ & $96 \%$ \\
\hline
\end{tabular}

The data in Table 2 indicates some striking enhancements in sensitivity, which are traced to non-orthogonal polarization retrievals. In the summer where the sun remains up 24 hours a day, the weak perpendicular polarization of the MPL is limited in range, but non-orthogonal polarization retrievals with CAPABL's parallel and $3^{\text {rd }}$ channel ( 45 degrees from both parallel and perpendicular) have greatly enhanced range. This enhanced ranged is due to the small difference in signal strengths between CAPABL's signals.

\subsection{MILLIMETER CLOUD RADAR}

A zenith pointing Ka-band $(35 \mathrm{GHz})$ single polarization Doppler cloud radar (millimeter cloud radar or MMCR) is deployed in the same location as CAPABL, which is well described by $[13,14]$. A comparison of data products is not as simple as with an MPL because radar Doppler parameters do not directly correspond to CAPABL data masks. An example of a simple comparison is given in Fig. 1 where CAPABL cloud boundaries are plotted over MMCR reflectivity data. The cloud boundaries on the given day, Feb. 27, 2016, show good agreement in 
a bulk sense. CAPABL seems to have better sensitivity to high thin ice clouds than does the radar, as is expected, but attenuation is visible between approximately 21 and 24 UTC where the cloud top boundary from CAPABL does not correspond to the end of data for the MMCR.

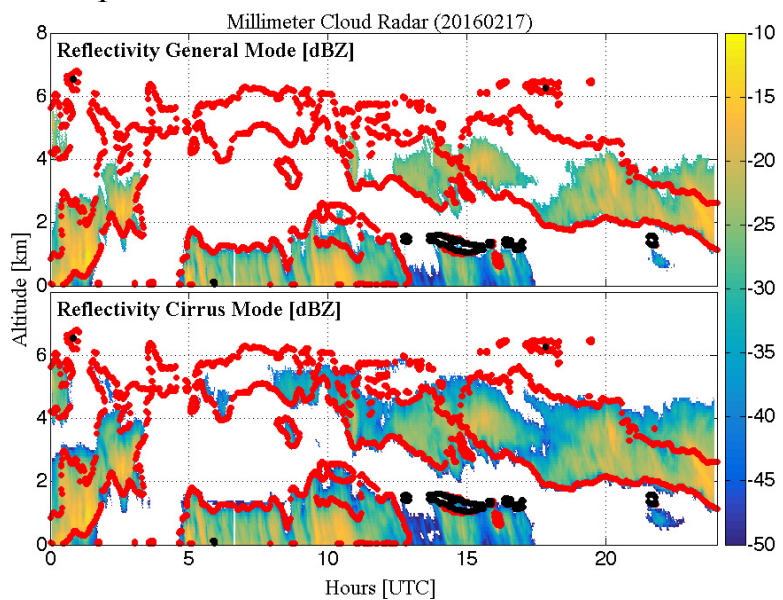

Figure 1: Radar reflectivity for its general mode and high sensitivity cirrus mode (top and bottom respectively). CAPABL derived cloud boundaries of ice and mixedphase clouds are given by the red and black respectively.

Radar data is compared to CAPABL data in a two-step process. First, as their temporal and spatial resolutions are not the same, MMCR data is incoherently averaged in time to a similar grid as CAPABL then linearly interpolated in 2 dimensions to CAPABL's grid. Second, the radar data is grouped by the lidar data mask, then the Doppler spectral characteristics are used to create a histogram, then a cumulative distribution function of the resulting data.

A four-month comparison from July to November 2016 of data is presented in Fig. 2, which includes more than 25 million MMCR and CAPABL data pixels with temporal and spatial resolution of approximately 80 seconds and 30 meters for which both instruments had nearly $100 \%$ uptime. The MMCR data of interest are reflectivity (integral of the Doppler spectrum), Doppler velocity (first moment of the Doppler spectrum), and Doppler spectral width (second moment of the Doppler spectrum). Also plotted is liquid water path, which is described in the next section.

The cumulative distribution functions indicate what percent of the data exists at or below a given level. For example, in Fig. 2, approximately 25\% of radar data identified by CAPABL as ice has a reflectivity less than $-25 \mathrm{dBZ}$, whereas approximately $75 \%$ of data identified as water has a reflectivity less than $-25 \mathrm{dBZ}$.

The expectations of this CAPABL/MMCR comparison should be clearly stated to put the resulting cumulative distribution functions into context. Broadly, at $35 \mathrm{GHz}$, the MMCR is more sensitive to ice than liquid water droplets via a diameter to the sixth $\left(\mathrm{D}^{6}\right)$ power scaling. By extension, the MMCR is more sensitive to liquid water droplets than clear air. One expects therefore, to see higher radar reflectivity for ice than liquid and less still for clear air. Furthermore, as ice is much larger, in diameter, at Summit than liquid water drops, one expects to see higher Doppler velocities (and given similar air motion, fall speeds) for ice. Finally, one expects Doppler spectrum width, which is dependent on a combination of microphysics and air motion, to be higher for liquid than ice or clear air due to turbulent motions that are typically associated with liquid phase clouds [15].

With these above expectations in mind, some reasonable and some striking results appear. The expected reflectivity and Doppler velocity cumulative distribution functions produce results as described above. Interestingly, Doppler velocities of ice crystals with a random orientation are higher than those with a preferential horizontal orientation. This is to be expected due to the drag forces that cause ice crystals to orient, but it is a useful indicator that polarization retrievals are reasonably separating randomly and preferentially oriented ice crystals. Additionally, the liquid spectral width curve shows interesting behavior both indicating large fractions of high spectral width particles but also a non-negligible $25 \%$ having much smaller spectral width than almost all of the ice pixels, indicating perhaps a separation between mixed phase and liquid only pixels.

\subsection{MICROWAVE RADIOMETER}

Comparing CAPABL data to that acquired by microwave radiometer (MWR) is also possible with the ICECAPS instrumentation. Two MWRs at Summit sample windows at 23.8, 31.4, 90, and $150 \mathrm{GHz}$. From these microwave brightness temperature observations, the liquid water path (LWP) is retrieved using optimal estimation [16]. 
These data are then linearly interpolated to the same time as CAPABL measurements for comparison.

CAPABL's data can be condensed into a column measurement based on the most radiatively important pixel type. A column is tagged as liquid if it contains any liquid. A column is tagged as ice if it contains ice and no liquid. A column can only be labeled as clear if no detectable cloud is present.

CAPABL column identifications can thus be used in a similar way to MMCR classification where MWR measurements are masked by CAPABL data, and then histograms and cumulative distribution functions are calculated for LWP for each mask type. This is also presented in Fig. 2 along with MMCR data previously described. Results show a very consistent agreement between CAPABL identifications of liquid clouds and MWR results.

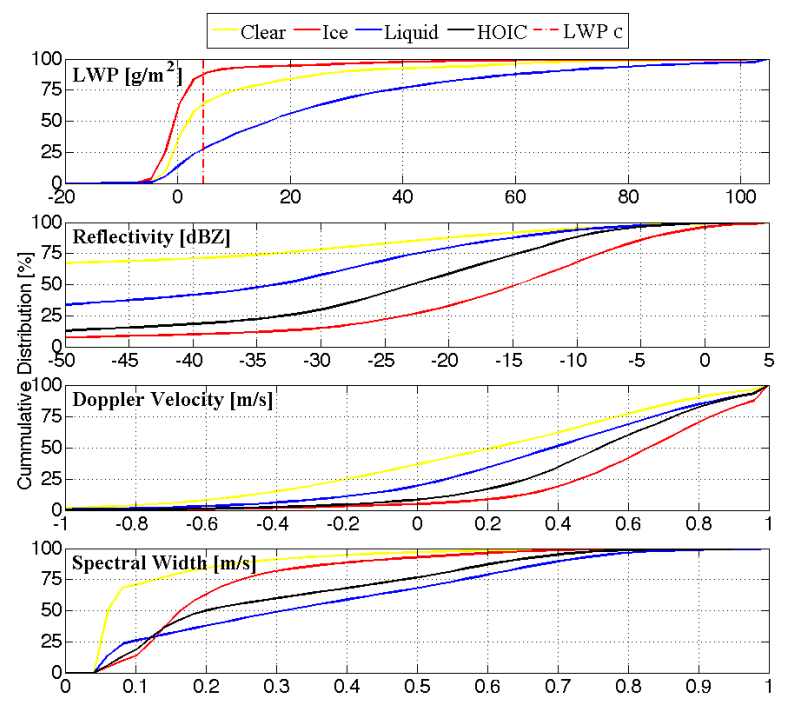

Figure 2: Cumulative distribution functions comparing MWR and MMCR data to CAPABL. The consistency of results is used in a broad sense to verify CAPABL's best estimate cloud mask.

\section{DISCUSSION AND CONCLUSIONS}

The data collected by CAPABL allows for an array of possible methods of calculation. Six possible methods were analyzed by [10] which showed dramatic differences in variables such as cloud fractional occurrence for different cloud phases as a function of processing type. This work has addressed that concern by combining the most critical processing types into a single cloud identification mask.

The process of creating this single mask however implicitly suggests a problem with validation. Validation of remote sensing equipment is challenging due to a lack of calibrated verification measurements. In this work, we choose to evaluate the CAPABL's phase identification algorithm by using ancillary remote sensing equipment to examine the consistency of retrievals. Here highly consistent results between CAPABL and collocated MPL, MMCR, and MWR over time periods from 1 month to 4 months are shown. One difficult is the validation of preferential orientation, because no sensors currently stationed at Summit are sensitive to such orientation except CAPABL. Differences in mean Doppler velocity measurements, related to particle fall speeds, demonstrate consistency with HOIC detection, but more work is needed to rigorously validate HOIC detections. More discussion is offered on the subject $[9,10,17]$.

\section{ACKNOWLEDGEMENT}

This material is based upon work supported by the NSF GRFP under Grant No. DGE 1144083 and NSF Grant No. AON 1303864 and 1314156.

\section{REFERENCES}

[1] Shupe, M. D., et al.: BAMS, 94, 2013.

[2] Gregory, J. M., et al.: Nature, 428, 2004

[3] Kjeldsen, K. K., et al.: Nature, 528, 2015.

[4] Bennartz, R., et al.: Nature, 49, 2013.

[5] Tan, I., et al.: Science, 352, 2016.

[6] Morrison, H., et al.: Nature, 5, 2012.

[7] Curry, J. A., et al.: J. of Climate, 9, 1996.

[8] Shupe, M.D., and J. M. Intrieri, J. Climate, 17, 2004

[9] Neely, R.R., et al.: JTECH, 2013.

[10] Stillwell, R.A., et al.: Atmos. Meas. Tech.

Discuss., in review, 2016.

[11] Turner, D.D., and R.G. Ellingson, Eds.:

Meteor. Monograph, No 57, AMS, 2016

[12] Campbell, J.R., et al.: JTECH, 19, 2002

[13] Moran, K.P., et al.: BAMS, 79, 1998.

[14] Clothiaux, E.E., et al.: JTECH, 16, 1999.

[15] Verlinde, J., et al., J. Geophys. Res. Atmos., 2013

[16] Cadeddu, M.P. et al., Atmos. Meas. Tech., 6, 2013

[17] Cole, S. et al.: Submitted to ACP, in review, 2016. 